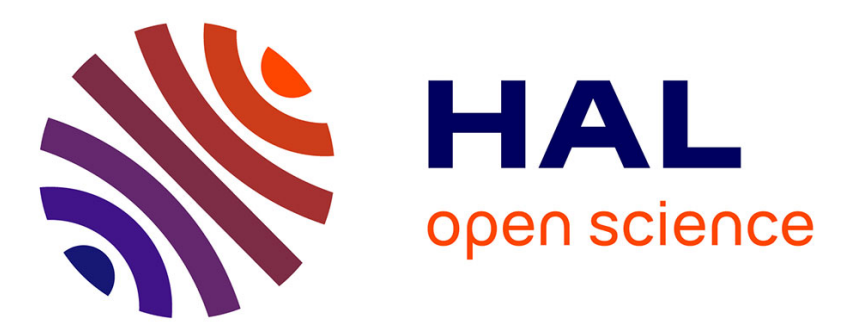

\title{
Synthesis of zinc sulfide multi-scale agglomerates by homogeneous precipitation-parametric study and mechanism
}

\author{
Mohamed Kamal Mekki Berrada, Frédéric Gruy, Michel Cournil
}

\section{To cite this version:}

Mohamed Kamal Mekki Berrada, Frédéric Gruy, Michel Cournil. Synthesis of zinc sulfide multi-scale agglomerates by homogeneous precipitation-parametric study and mechanism. Journal of Crystal Growth, 2009, 311 (8), pp.2459-2465. 10.1016/j.jcrysgro.2009.01.138 . hal-00411031

\section{HAL Id: hal-00411031 \\ https://hal.science/hal-00411031}

Submitted on 25 Aug 2009

HAL is a multi-disciplinary open access archive for the deposit and dissemination of scientific research documents, whether they are published or not. The documents may come from teaching and research institutions in France or abroad, or from public or private research centers.
L'archive ouverte pluridisciplinaire HAL, est destinée au dépôt et à la diffusion de documents scientifiques de niveau recherche, publiés ou non, émanant des établissements d'enseignement et de recherche français ou étrangers, des laboratoires publics ou privés. 


\title{
Synthesis of zinc sulfide multi-scale agglomerates by homogeneous precipitation-parametric study and mechanism
}

MeKKi-BerRAdA, M.K. ; GRUY, F. ; COURNIL, M. (*)

(1) Département GENERIC (Géochimie, ENvironnement, Ecoulement, Réacteurs Industriels et Cristallisation), Centre SPIN. LPMG UMR CNRS 5148. Ecole Nationale Supérieure des Mines de Saint-Etienne 158, Cours Fauriel. 42023 Saint-Etienne Cedex 2.

(*) cournil@emse.fr

Keywords:

A1. Crystal morphology; Multi-scale agglomerates; A2. Industrial crystallization; Homogeneous precipitation; B1. Zinc sulfide

PACS classification codes:

64.70.dg; 64.70.kp; 87.15.nr; 74.62.Bf; 75.50.Tt

\begin{abstract}
When zinc sulfide is prepared by homogeneous precipitation from an acid solution of zinc sulfate and thioacetamide in a stirred reactor, it is generally obtained in the form of four-scale agglomerates. This paper is intended to progress in the understanding of the nature of the construction stages of these agglomerates. For this, successive size scales are studied as a function of several operating parameters: $\mathrm{pH}$, concentration in reactants, temperature and stirring rate. Microscopic characterization is performed at different times of the precipitation process. The successive steps of construction of the multi-scale structure aggregates are discussed in the context of the model of Eshuis and Koning, Coll. Polymer Sci. 272 (1994) 1240 and Eshuis et al. Colloid and Surfaces, Physicochemical and Engineering Aspects 151 (1999) 505 .
\end{abstract}

\section{Introduction}

Powder materials in the form of particle agglomerates are commonly produced by precipitation processes. Sometimes these particles are themselves made up of smaller crystals. This particular morphology of multi-scale agglomerates is of potential great interest for industrial applications because the same solid product is liable to present different size scales with different associated properties or functions.

This work consists first of a systematic experimental study of the precipitation of multi-scale agglomerates in a model system. Its final objective, however, is to advance the understanding of the formation mechanism of these multiple agglomerates in order to be able to control their synthesis and give them the desired end-use properties.

In this work, zinc sulfide, which is generally obtained in the form of four-scale agglomerates, was chosen as model product. Another reason for this choice is that it can be produced by homogeneous precipitation.

In homogeneous precipitation, the initial medium is an inert solution of one of the precipitation reagents (A) and another product (C) which decomposes when temperature increases and slowly and progressively releases the other precipitation reagent (B). Consequently, (B) is homogeneously distributed in the bulk of the solution. The resulting supersaturation is low and uniform. Mixing effects are negligible. Thus the medium composition is better controlled, and so is the reproducibility. Homogeneous precipitation is 
considered as one of the most relevant methods to obtain particles of homogeneous size and morphology [1] and [2].

\section{Basic concepts}

Experimental works on $\mathrm{ZnS}$ homogeneous precipitation use an acid solution of zinc acetate, nitrate, chloride or sulfate and thioacetamide $\left(\mathrm{CH}_{3} \mathrm{CSNH}_{2}\right)$ as initial medium [3], [4], [5], [6], [7], [8] and [9]. Thiocetamide (TAA) is currently used as a hydrogen sulfide source in different organic syntheses. In acid medium and above $60{ }^{\circ} \mathrm{C}$, indeed, TAA decomposes according to the following reaction:

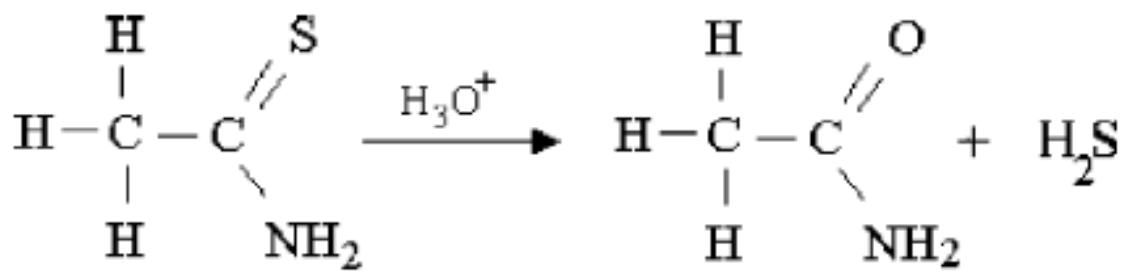

This irreversible reaction which is catalyzed by hydronium ions releases $\mathrm{H}_{2} \mathrm{~S}$ in a homogeneous way in the solution [10] and [11]. Best decomposition conditions are obtained for $\mathrm{pH}<2$. Sulfide $\left(\mathrm{S}^{2-}\right)$ anions coming from $\mathrm{H}_{2} \mathrm{~S}$ then react with $\mathrm{Zn}^{2+}$ cations to give precipitated $\mathrm{ZnS}$ :

$\mathrm{Zn}^{2+}+\mathrm{S}^{2-} \rightarrow \mathrm{ZnS}$

In most works, the effects of stirring, $\mathrm{pH}$, concentration, viscosity and the presence of cations were the subject of essentially qualitative studies. In particular the relatively basic geometry of the reactors did not allow a real determination of the influence of stirring. Only Eshuis and Koning [8] and Eshuis et al. [9] performed a systematic study of the dependence of the agglomerate morphology on the experimental parameters and proposed the most advanced mechanism of $\mathrm{ZnS}$ multi-scale agglomeration ever presented so far. The four steps of the mechanism (1)-(4) are represented in Fig. 1; they are supposed to give rise to the four successive size scales and agglomeration structures (I)-(IV).

* Step 1 consists of nucleation-growth of $20-80 \mathrm{~nm}$ crystallites from highly supersaturated solutions.

* In step 2, Brownian agglomeration gives birth to chains of about twenty crystallites which were observed as independent objects by Ref. [7].

- During step 3, these chains agglomerate and form spherical porous particles of 1$5 \mu \mathrm{m}$ in diameter.

* Step 4 consists in the formation of large agglomerates of these spherical particles.

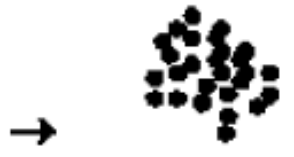

(1)

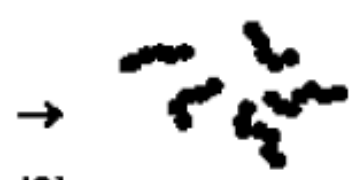

(2)

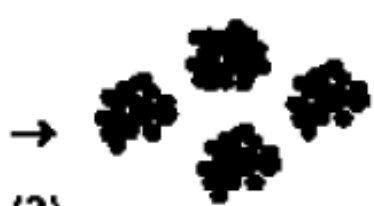

(3)

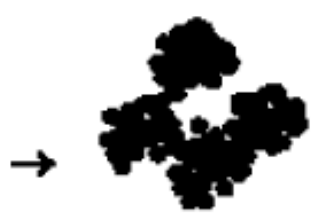

(4)

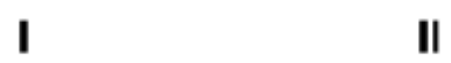

III

Fig 1: Representation of the steps of a 4-scale agglomeration according to Refs. [8] and [9] 
Other mechanisms exist, they are indeed more quantitative, but they are however not so precise concerning the morphological aspects [12] and [13].

Some general points however remain poorly understood: why are such different size scales obtained and what is their origin? Why are so narrowly dispersed particles and so regular agglomerates formed whereas most of the processes are a priori random and simultaneous?

To try to answer these questions a systematic experimental study in reproducible conditions seems necessary. To this aim we designed an experimental set-up which is presented in the next section. The precipitation is operated in a standard stirred and thermostated reactor which allows us to study the effects of stirring rate, $\mathrm{pH}$, reactant concentration and temperature on the morphology of the zinc sulfide agglomerates. Some of these results have been presented elsewhere in an experimental paper [14]. They are considered here in the context of a modeling approach and completed with new observations concerning the evolution of the agglomerate morphology throughout the precipitation process. Thanks to all these results a new mechanism is proposed for the formation of the zinc sulfide multi-scale agglomerates.

\section{Experimental procedure}

The set-up is composed of a main reactor and of an auxiliary reactor (Fig. 2). The main reactor in which precipitation proceeds has a maximum volume of $3 \mathrm{~L}$; it is double-jacketed, mechanically agitated using a $45^{\circ}$ four-blade propeller (stirring rate $N$ ) (diameter $5 \mathrm{~cm}$ ) and provided with 4 Teflon baffles. Excess of gaseous hydrogen sulfide is entrained by a slow nitrogen flow-rate $\left(1 \mathrm{~N} \mathrm{~L} \mathrm{~h}^{-1}\right)$ to the auxiliary reactor in which it is absorbed by a $0.5 \mathrm{M}$ soda aqueous solution.

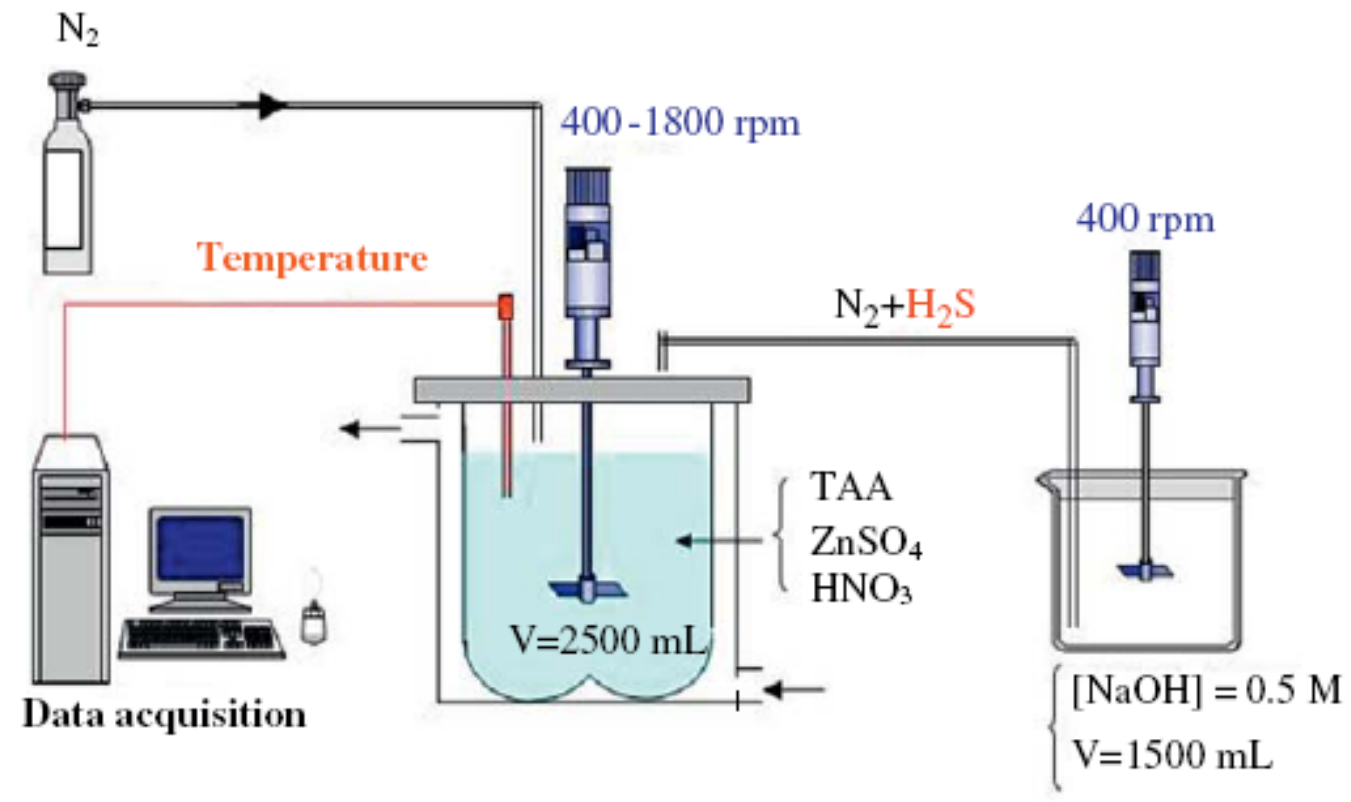

Fig 2: Experimental set-up.

Two solutions of $1250 \mathrm{~mL}$ of thioacetamide and zinc sulfate are prepared independently. Concentration in zinc sulfate is kept constant in all experiments whereas TAA concentration is varied. Nitric acid is added in the zinc sulfate solution to adjust the global $\mathrm{pH}$ at the desired value. Reactant solutions are then introduced into the main reactor. Liquid height is equal to the reactor diameter as recommended in crystallization studies. This corresponds to a liquid volume of $2.5 \mathrm{~L}$. The main reactor is initially thermostated at $25{ }^{\circ} \mathrm{C}$. Under such conditions, initial mixture is inert. To obtain sufficiently high and rapid decomposition of TAA, heating 
up at $1.6^{\circ} \mathrm{C} \mathrm{min}-1 /$ to the working temperature (higher than $60^{\circ} \mathrm{C}$ ) is operated. Temperature is then maintained at constant value for about $3 \mathrm{~h}$. Precipitation proceeds as proved by visually observed turbidity. Then, temperature is decreased to ambient temperature by natural cooling. Zinc sulfide suspension samples can be collected, withdrawn and characterized both at the end of the experiment and at earlier stages.

Suspension samples are filtered at $0.45 \mu \mathrm{m}$ then washed five times with distilled water and at last dried at $50^{\circ} \mathrm{C}$ for $12 \mathrm{~h}$. The so-obtained samples can be observed by scanning electronic microscopy (JSM 6500 FEG) and characterized by X-ray diffractometry (Siemens D5000) and laser scattering particle sizing (Malvern Mastersizer 2000). The light scattering experiments carried out with the original reaction mixture or with dried and resuspended $\mathrm{ZnS}$ powder give the same particle size distribution. This tends to prove that the collected powder is not damaged by the washing and drying treatments.

\section{Results}

General features

Zinc sulfide currently presents two crystallographic varieties, hexagonal wurtzite and cubic sphalerite (or blende). In this work, only sphalerite was observed.

Fig. 3a shows a general view of the $\mathrm{ZnS}$ particles when removed at the end of the experiment. Fig. $3 \mathrm{~b}-\mathrm{d}$ clearly highlights the successive scales of the agglomerates. Scale 1 certainly corresponds to crystallites. These crystallites seem to be themselves grouped in oval structures in form of "caterpillars" (scale 2). Scale 3 corresponds to spheres which are themselves gathered in relatively ramified agglomerates (scale 4). This succession of structures is observed practically for all the experiments which are described here.

a

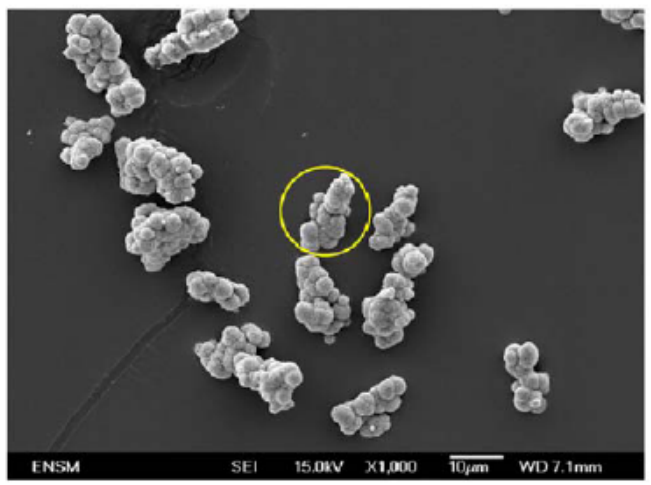

C

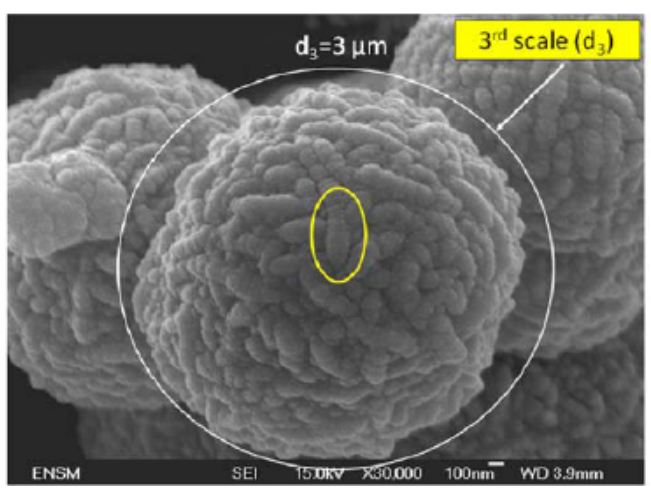

b

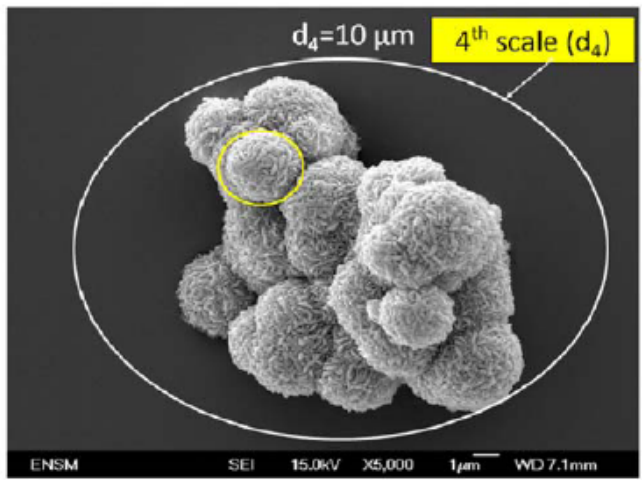

d

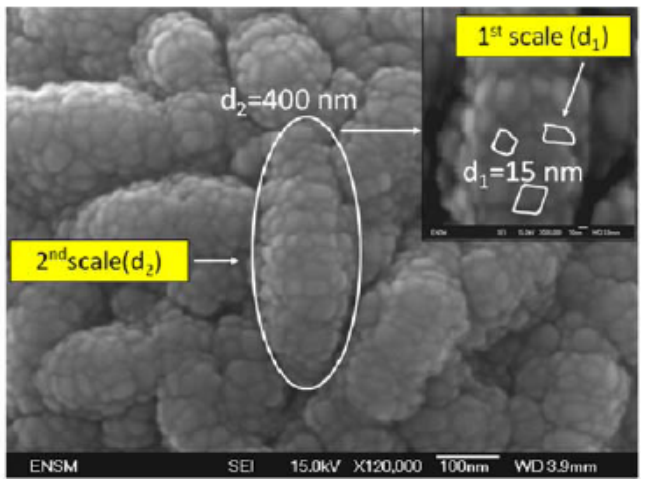

Fig 3: ZnS agglomerate morphology ([TAA] $=0.5 \mathrm{~mol} \mathrm{~L}^{-1} ; \mathrm{pH}=1.5 ;\left[\mathrm{ZnSO}_{4}\right]=0.025 \mathrm{~mol} \mathrm{~L}^{-1} ; \mathrm{T}=8 \mathrm{O}^{\circ} \mathrm{C}$; no stirring). (a) General view, (b) fourth scale, (c) third scale and (d) second and first scales. 
Parametric study of the final agglomerate scale size dependence

Determining the influence of the main operating parameters on the morphology of the agglomerates is a crucial step for the comprehension of their construction. To this aim, a systematic study of influence of $\mathrm{pH}$, TAA concentration, temperature $T$ and stirring rate $N$ has been performed. These parameters have been chosen for the following reasons. TAA decomposition is indeed catalyzed by $\mathrm{H}_{3} \mathrm{O}^{+}$ions, thus only the narrow $\mathrm{pH}$-range [0.5-2.5] has been investigated. TAA concentration indirectly influences supersaturation via produced $\mathrm{H}_{2} \mathrm{~S}$ and $\mathrm{S}^{2-}$ (literature reports weak influence of $\mathrm{Zn}^{2+}$ ions). Temperature acts at different levels (TAA decomposition, nucleation-growth).

The stirring rate is known to strongly affect aggregation and possibly crystal growth. Variation ranges of these different parameters are given in Table 1.

Table 1: Variation range of $p H,[T A A], \mathrm{T}, N$.

\begin{tabular}{|cc|}
\hline Operating parameter & Variation range \\
\hline $\mathrm{pH}$ & $0.5^{-2.5}$ \\
\hline Concentration in TAA $\left(\mathrm{mol} \mathrm{L}^{-1}\right)$ & $0.05^{-0.8}$ \\
\hline$[\mathrm{TAA}] /[\mathrm{Zn}]$ & $1-16$ \\
\hline Temperature $\left({ }^{\circ} \mathrm{C}\right)$ & $65^{-80}$ \\
\hline Stirring rate $(\mathrm{rpm})$ & $400-1800$ \\
\hline
\end{tabular}

An overview of the results of this systematic study is shown in Table 2. The intensity of the influence of a given parameter on crystallite size (scale 1: about $20 \mathrm{~nm}$ ), sphere size (scale 3:1-6 $\mu \mathrm{m}$ ) and largest agglomerate size (scale 4:6 to $50 \mu \mathrm{m}$ ) is symbolized by (+++) (strong), $(++)$ (average), $(+)$ (weak or dubious). Scale $2(100-150 \mathrm{~nm})$ is not considered for the moment, due to the complexity of its morphology. Scale 1 size was deduced from XRD peak width. Direct measurement on SEM photographs confirmed the accuracy of this determination method. Scale 3 is directly observed on SEM pictures. Scale 4 can also be obtained from SEM photographs. However, it is more easily given by D [3] and [4] particle size measurements and both methods yield similar results.

Table 2: Effect of parameters on crystallite and agglomerate size (scales).

\begin{tabular}{|ccccc|}
\hline Scale & pH & [TAA] & T & N \\
\hline 1 & +++ & + & ++ & + \\
\hline 2 & +++ & +++ & +++ & + \\
\hline 3 & + & ++ & +++ & +++ \\
\hline
\end{tabular}

Our results can be compared profitably with some of those obtained by Refs. [8] and [9] (Table 3).

Table 3: Effect of parameters on scale transition (as reported by Refs. [8,9]).

\begin{tabular}{c|cc|}
\hline Transition & pH & Stirring rate \\
\hline I $\rightarrow$ II & +++ & + \\
\hline II $\rightarrow$ III & +++ & ++ \\
III $\rightarrow$ IV & +++ & +++ \\
\hline
\end{tabular}

Although these authors express the effect of the parameters on different transitions, certain similarity exists with our results, except for the $\mathrm{pH}$ effect on the largest sizes.

We can now examine the detailed effect of each parameter on different scales.

(a) $\mathrm{pH}$ effect is significant on the smallest scales, particularly at $\mathrm{pH}<1$. Crystallite diameter is a decreasing function of $\mathrm{pH}$. Sphere diameter (scale 3) increases then decreases with pH (Fig. 4). For the sake of simplicity, scale size has been made dimensionless through division by the mean diameter value for each scale. Behavior at pH 0.5 (Fig. 5a) is 
uncommon; it is characterized by a sand-rose morphology (scale 3) whose petals (scale 2) are layers made up of crystallites whereas, at higher $\mathrm{pH}$ (2.5), the usual structure is retrieved (Fig. 4b).

(b) Concentration in TAA has a significant influence on scales 3 and 4, particularly at low TAA excess. Corresponding diameters are increasing functions of [TAA]; it is worthwhile mentioning that the behavior of scales 3 and 4 is identical (Fig. 6). This tends to prove that, at first approximation, the number of spheres in large agglomerates and the morphology of the latter do not depend on concentration in [TAA]. This is confirmed by microphotograph observations.

(c) Temperature has an influence on all scales (Fig. 7). Diameters appear as increasing functions of temperature. The different plots seem to be homothetic.(d) Stirring rate has an effect only on the largest scale. This wellknown influence is represented by a relation between the agglomerate diameter and the Kolmogorov scale which characterizes the size of the smallest eddies in a turbulent flow [15]

Fig. 8 shows the variation of the agglomerate mean size against the Kolmogorov microscale $\lambda_{K}=\left(v^{3} / \varepsilon\right)^{1 / 4}$. In this relation, $v$ is the kinematic viscosity of the liquid phase and $\varepsilon$ is the turbulent energy dissipation rate. A linear relation is clearly visible.

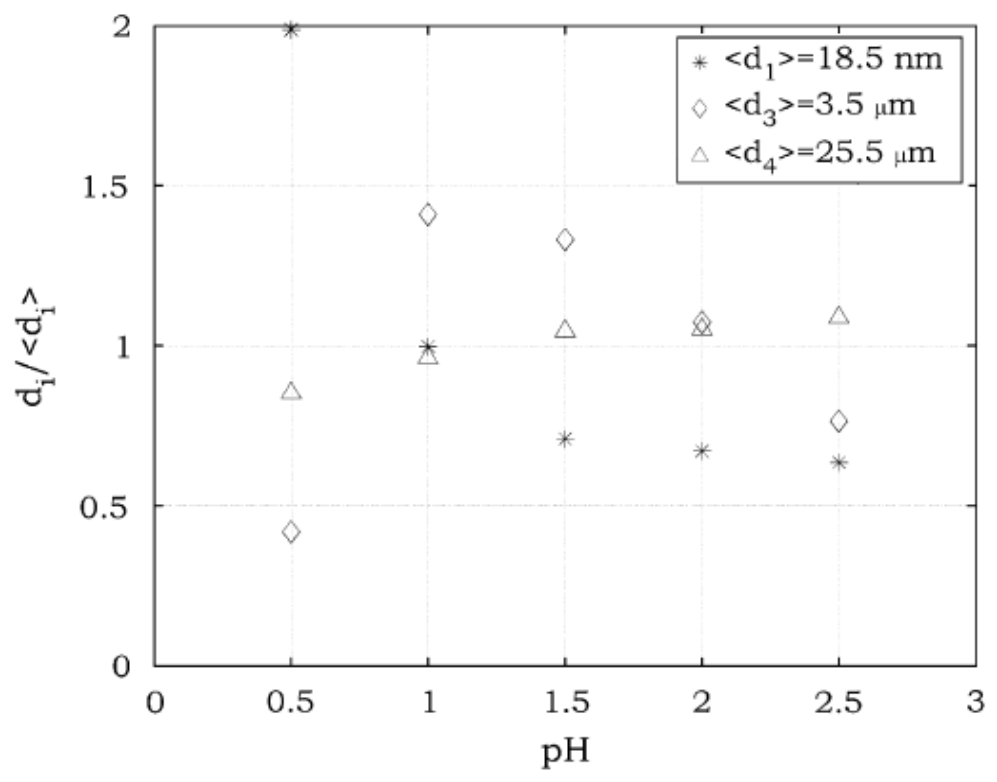

Fig 4: Dimensionless diameter of scales 1, 3 and $4\left(d_{1} /\left\langle d_{1}\right\rangle, d_{3} /\left\langle d_{3}\right\rangle, d_{4} /\left\langle d_{4}\right\rangle\right)$ vs. $p H$; $[T A A]=0.1 \mathrm{~mol} \mathrm{~L}^{-1},\left[\mathrm{ZnSO}_{4}\right]=0.025 \mathrm{~mol} \mathrm{~L}-1, N=700 \mathrm{rpm}$ and $T=80^{\circ} \mathrm{C}$. 
a

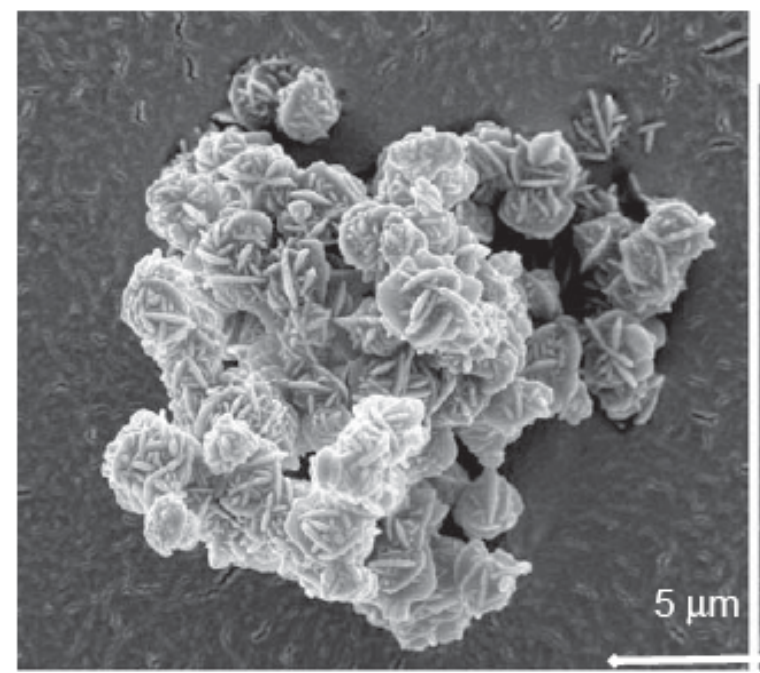

b

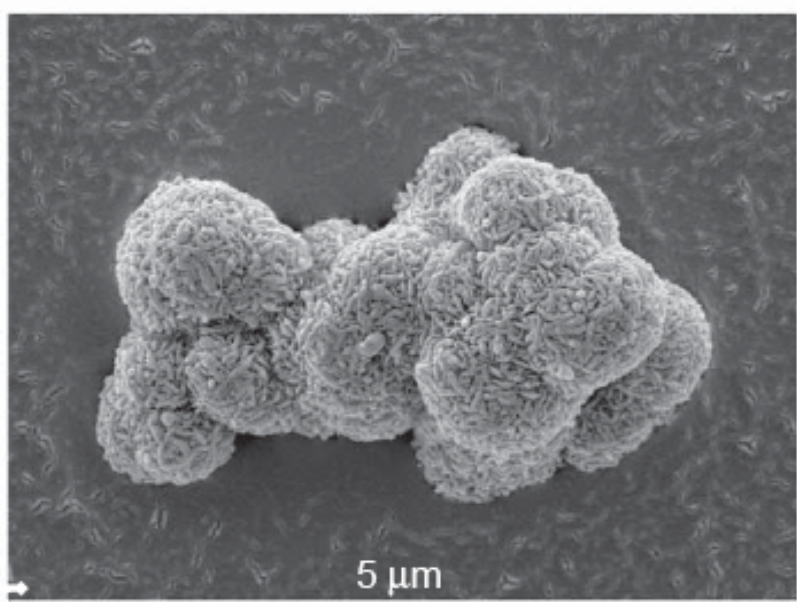

Fig 5: Morphology of the agglomerates at $p H=0.5$ (a) and $p H=2.5$ (b) $[T A A]=0.1 \mathrm{~mol} \mathrm{~L}^{-1}$, $\left[\mathrm{ZnSO}_{4}\right]=0.025 \mathrm{~mol} \mathrm{~L}^{-1}, \mathrm{~N}=700 \mathrm{rpm}$ and $\mathrm{T}=8 \mathrm{O}^{\circ} \mathrm{C}$.

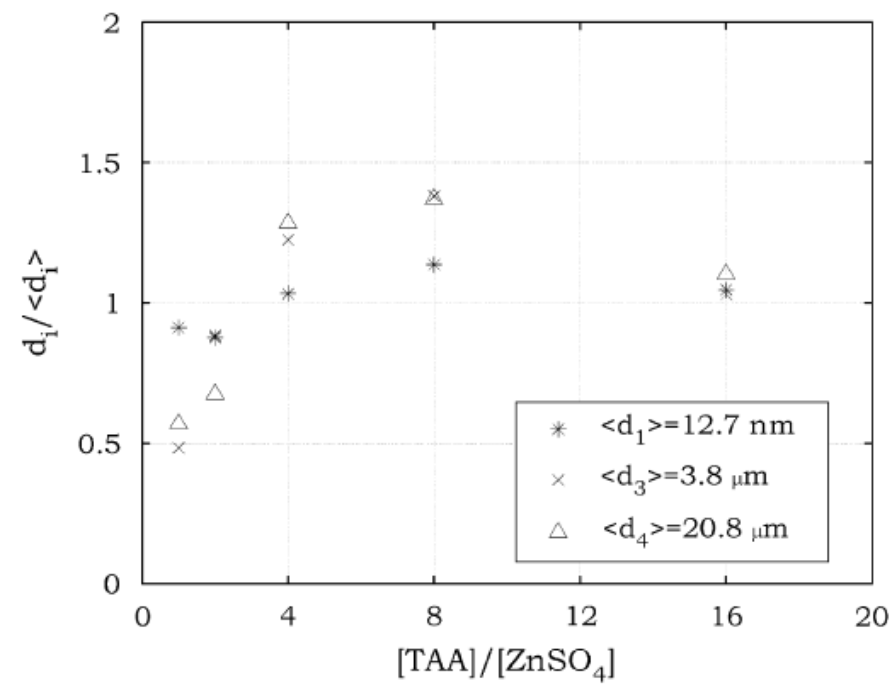

Fig 6: Dimensionless diameter of scales 1, 3 and $4\left(d_{1} /\left\langle d_{1}\right\rangle, d_{3} /\left\langle d_{3}\right\rangle, d_{4} /\left\langle d_{4}\right\rangle\right)$ vs. [TAA]/[ZnSO $\left.{ }_{4}\right]$ ratio; $\left[\mathrm{ZnSO}_{4}\right]=0.025 \mathrm{~mol} \mathrm{~L}^{-1}, \mathrm{pH}=1.5, \mathrm{~T}=80^{\circ} \mathrm{C}$ and $\mathrm{N}=700 \mathrm{rpm}$.

Agglomerate morphology at earlier stages of the precipitation process

Zinc sulfide precipitation is supposed to start as soon as a sufficient supersaturation level is reached, i.e., a few minutes after the beginning of TAA decomposition. The thermal protocol which is adopted here means after $40-50$ min of heating. Previous photographs (Fig. 3, Fig. 5 and Fig. 8) are relative to "final" agglomerates, i.e. to samples withdrawn from the solution after $3 \mathrm{~h}$ of experiments or more.

Given that in present semi-batch configuration, experimental parameters such as $\mathrm{pH}$ and supersaturation are not kept constant, different steps of the precipitation process, nucleation, growth, agglomeration do not take place in steady conditions. Thus information on the experimental conditions throughout the whole experiment would be necessary to propose a mechanism for the precipitation process especially as regards its morphological aspects. In the absence of this data, observation of $\mathrm{ZnS}$ samples withdrawn at earlier times from the suspension is particularly useful. 


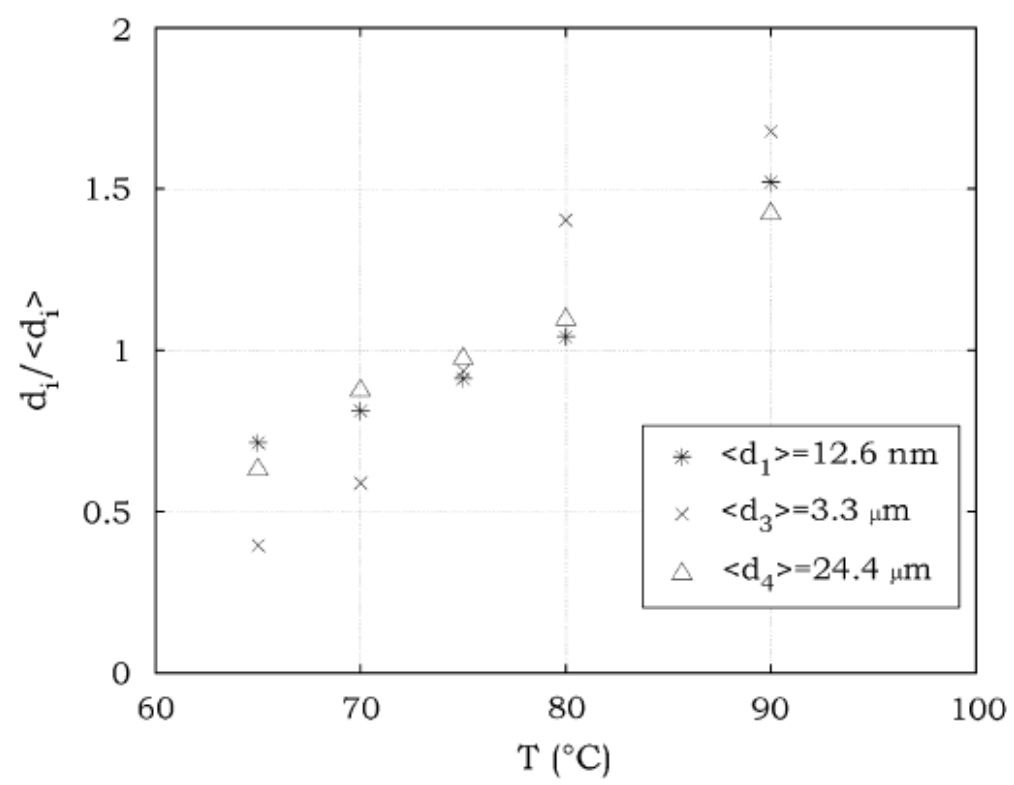

Fig 7: Different scale sizes vs. temperature T; $p H=1.5,[T A A]=0.1 \mathrm{~mol} \mathrm{~L}^{-1},\left[\mathrm{ZnSO}_{4}\right]=0.025 \mathrm{~mol} \mathrm{~L}^{-1}$ and $\mathrm{N}=700$ rpm.

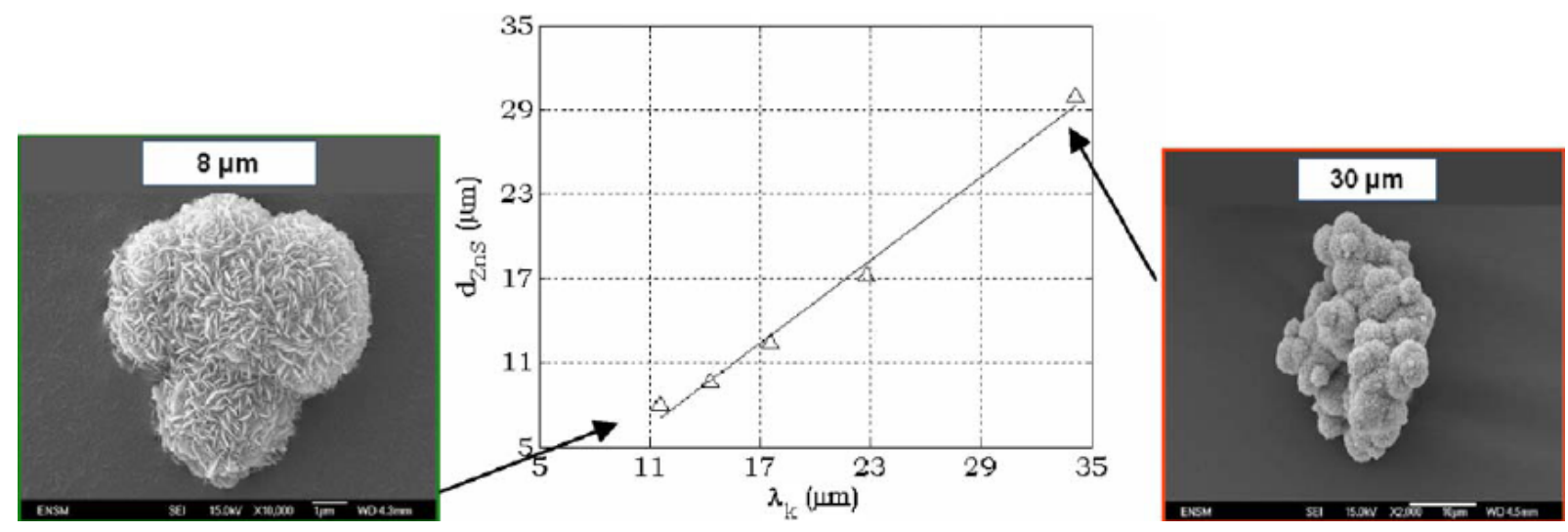

Fig 8: Agglomerate diameter against Kolmogorov scale; $\quad p H=1.5, \quad[T A A]=0.1 \mathrm{~mol} \mathrm{~L}^{-1}$, $\left[\mathrm{ZnSO}_{4}\right]=0.025 \mathrm{~mol} \mathrm{~L}^{-1}$ and $\mathrm{T}=80^{\circ} \mathrm{C}$.

Sample removal, however, is not possible at times earlier than 25 min because of the very low mass and small size of the precipitated particles. In Fig. 9, we present microphotographs of the same magnification $(\times 25,000)$ of $\mathrm{ZnS}$ samples removed at different times and prepared under the following initial conditions: $\mathrm{pH}=1.5$, $[\mathrm{TAA}]=0.1 \mathrm{M},\left[\mathrm{ZnSO}_{4}\right]=0.025 \mathrm{M}, N=700 \mathrm{rpm}$ and $T=80^{\circ} \mathrm{C}$. Time zero corresponds to the beginning of TAA decomposition (onset of supersaturation).

It clearly appears from these pictures that

* multi-scale structure appears relatively early; in particular, scale 3 (sphere) is observed only after 30 min;

* scale 2 (caterpillars) is never observed separately. It seems indeed to be structured on the spot;

* development of large agglomerates seems to be globally homothetic. 

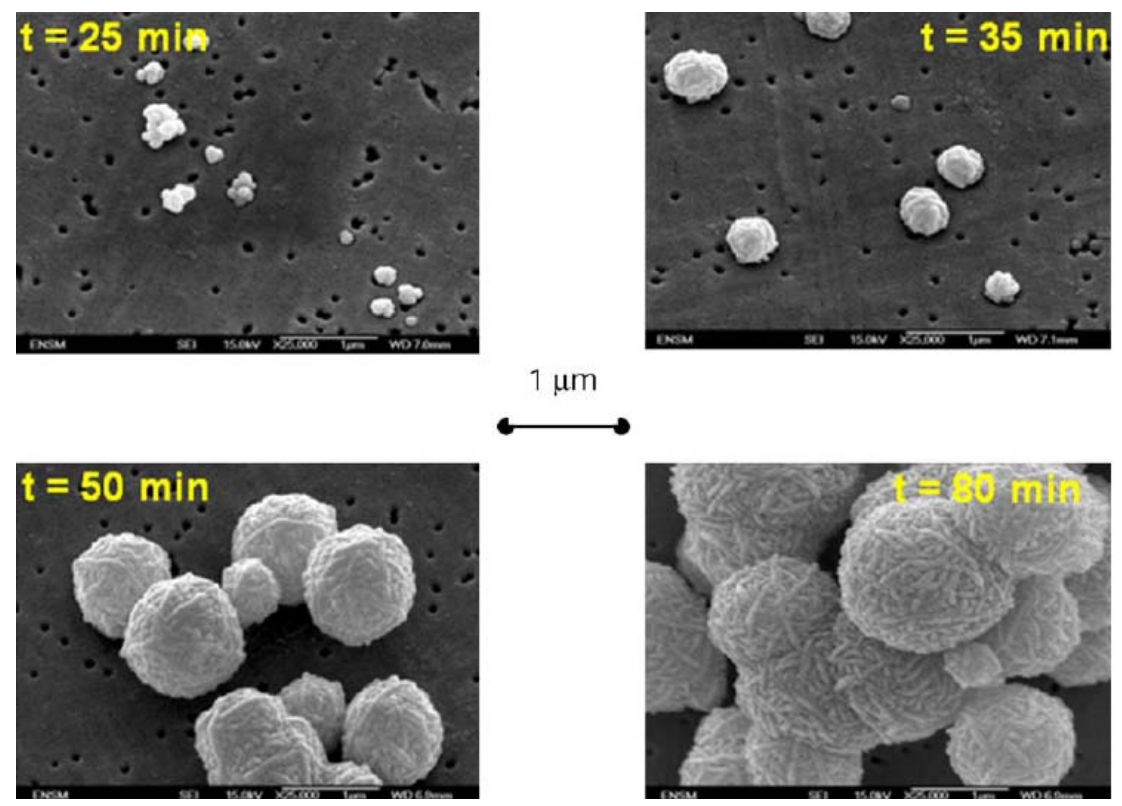

Fig 9: Morphology of $\mathrm{ZnS}$ samples removed at different precipitation times.

\section{Discussions}

The main objective of this work was to make progress in understanding the construction mechanism of zinc sulfide multi-scale agglomerates. This means in particular to be able to answer different questions such as: do the various structures follow one another in time and how? How is it possible to discern the hydrodynamic and chemical effects?

Chemical effects appear via the influence of $\mathrm{pH}\left(\left(0.5^{-2.5}\right)\right.$ range), TAA concentration and temperature.

* At the lowest $\mathrm{pH}$ values, TAA decomposition is more active and releases more $\mathrm{H}_{2} \mathrm{~S}$. However, due to the acid-base equilibria, concentration in sulfide ions is lowered. Consequences on supersaturation are difficult to predict. For the moment, we do not have a clear understanding of the trends observed in Fig. 4. Fig. 5a proves however that some structures (sand-rose) of the $\mathrm{ZnS}$ agglomerates are clearly due to crystal growth.

* Higher concentrations in TAA are expected to increase nucleation and growth rates via the supersaturation value; this effect is actually observed on scale 3 and homothetically on scale 4 (Fig. 6). Relative invariance of scale 1 is more surprising;

* Temperature probably has an acceleration effect on all the processes, TAA decomposition, $\mathrm{ZnS}$ nucleation and growth; this explains the global homothetic influence mentioned above. Moreover, Fig. 9 seems to clearly show us that the multi-scale structure is present from the early stages of the precipitation.

Now we propose to examine the possible links between different agglomerate size scales and the hydrodynamic scales. David et al. [16] proposed such an approach for three-scale agglomerates. In their work, crystallites (scale 1) are produced by intensive nucleation followed by growth. They rapidly aggregate to give scale 2 particles. This agglomeration step proceeds according to Brownian dynamics. Scale 2 is close to the Batchelor scale $\lambda_{B}=\left(v D^{2} / \varepsilon\right)^{1 / 4}$ which marks the limit between Brownian and laminar shear flow agglomeration $(D$ denotes Brownian diffusivity). Laminar agglomeration takes place in fact in turbulent eddies whose 
size is equal to the Kolmogorov scale $\lambda_{K}=\left(v^{3} / \varepsilon\right)^{1 / 4}$ and is close to the observed size of the largest agglomerates (scale 3).

In the case of $\mathrm{ZnS}$ precipitation, we proved in Fig. 8 that the Kolmogorov scale somewhat determines the largest agglomerate size. Batchelor size is about $200 \mathrm{~nm}$ in our case. Its intervention in the agglomeration dynamics is not evident. Actually, hydrodynamics is able to account for three successive agglomerate scales, however not for four as assumed within the mechanism of Eshuis and Koning [8] and Eshuis et al. [9].

We think that the existence of independent scale 2 structures (elongated ellipsoids) is questionable. This conjecture is supported by the following elements:

* Contrarily to the results reported in Ref. [7], scale 2 agglomerates are never observed alone on the photographs nor detected in different light scattering experiments (not presented here for lack of space; details can be found in Ref. [17]). In particular, in situ turbidity measurements clearly show the nucleation step of scale 1 crystallites. They however do not evidence independent scale 2 agglomerates, which should be accessible to this technique. Laser beam scattering is sensitive to scale 3 and scale 4 particles. Scale 2 agglomerates are not detected either. They would be however at the lower detection range of this technique.

- Scale 2 onset involves for sure aspects more chemical than hydrodynamic. We are more particularly considering oriented agglomeration and crystal growth. As aforesaid, picture 5a for instance clearly shows structure due to crystal growth (sand-rose).

* In Fig. 9 and on pictures which are not presented here, structure 2 seems to emerge gradually from the surface of the spheres and could constitute intrinsic morphological structures of the scale 3 spherical agglomerates.

In the absence of independent scale 2 particles, the model of Eshuis and Koning [8] and Eshuis et al. [9] does not hold any more and should be replaced by an alternative formulation. Neither agglomeration step: crystallite (scale 1) $\rightarrow$ caterpillar (scale 2), nor step: caterpillar (scale 2) $\rightarrow$ sphere (scale 3) would take place in reality, whereas steps: crystallite (scale 1) $\rightarrow$ sphere (scale 3) and of course spheres $\rightarrow$ large agglomerate would be the predominant processes.

Given final size of the spheres $(1-3 \mu \mathrm{m})$ and of the crystallites $(40 \mathrm{~nm})$ on the one hand, and Batchelor scale value $(200 \mathrm{~nm})$ on the other hand, crystallite agglomeration follows Brownian (perikinetic) dynamics and sphere agglomeration laminar (orthokinetic) dynamics.

\section{Conclusions}

In the (0.5-2.5) pH-range, zinc sulfide homogeneous precipitation from thioacetamide-zinc sulfate solutions produces agglomerates in which four scales can be discerned: the smallest one (scale 1) is essentially sensitive to $\mathrm{pH}$ and temperature, the second and third scale depend on temperature, $\mathrm{pH}$, concentration in TAA, the largest scale (scale 4) depends on concentration in TAA, temperature and stirring rate.

These results confirm those obtained by Eshuis and Koning [8] and Eshuis et al. [9] while also being more complete and quantitative.

Microphotographs of $\mathrm{ZnS}$ samples withdrawn at different precipitation times have led us to propose considering an alternative mechanism to Eshuis and Koning [8] and Eshuis et al. [9] theory because the existence of independent scale 2 agglomerates is not confirmed by our experiments.

The main elements of this mechanism are as follows: 
* Crystallites (scale 1) appear from sudden nucleation and growth of a large swarm of nuclei created in high supersaturation conditions.

* Scale 3 results from crystallite agglomeration.

* Scale 2 is probably not due to agglomeration itself, but to oriented integration of crystallites into the spherical particles.

* Scale 4 is imposed by hydrodynamics (either zero agglomeration efficiency or strong fragmentation beyond Kolmogorov scale).

Model improvement would require knowledge of variation of the experimental system's characteristics, especially supersaturation, throughout the precipitation process.

\section{References}

[1] R. Willams, P.N. Yocom, F.S. Stofko, J. Coll.Inter. Sci. 106 (1985) 388.

[2] H. Hofmann, Particle synthesis in condensed phases. Eastern Michigan University USA, June 2004. ACS PRF Summer School on Nanoparticle Materials.

[3] E. Butler, D.G. Peters, E.H. Swift, Anal. Chem. 30 (1958) 1379.

[4] D.F. Bowersox, D.M. Smith, E.H. Swift, Talanta 3 (1960) 282.

[5] G. Chiu, J. Coll. Inter. Sci. 83 (1981) 309.

[6] D.M. Wilhelmy, E. Matijevic, J. Chem. Soc. 80 (1984) 563.

[7] A. Celikkaya, M. Akinc, J. Am. Ceram. Soc. 73 (1990) 245.

[8] A. Eshuis, C.A.J. Koning, Coll. Polymer Sci. 272 (1994) 1240.

[9] A. Eshuis, G.R.A. Van Elderen, C.A.J. Koning, Colloid Surfaces, Physicochemical Eng. Aspects 151 (1999) 505 .

[10] T. Sugimoto, Surfactant Science Series, vol. 92, Marcel Dekker, New York, 2000, p. 190.

[11] O. M Peeters, C.J. de Ranter, J. Chem. Soc. Perkin Trans. 2 (1974) 1832.

[12] V. Privman, D.V. Goia, J. Park, E. Matijevic, J. Coll. Interf. Sci. 213 (1999) 36.

[13] S. Libert, V. Gorshkov, D. Goia, E. Matijevic, V. Privman, Adv. Coll. Interf. Sci. 100 (2003) 169.

[14] M.K. Mekki-Berrada, F. Gruy, T. Kuntzsch, M. Cournil, Ceramic Transactions, V 172, Ceramic Nanomaterials and Nanotechnology IV Proceedings of the 107th Annual Meeting of The American Ceramic Society, 2006, p. 3.

[15] P. Sessiecq, F. Gruy, M. Cournil, J. Crys. Growth 208 (2000) 555.

[16] R. David, F. Espitalier, A. Cameirao, L. Rouleau, Kona 21 (2003) 40.

[17] M.K. Mekki-Berrada, Synthèse d'agglomérats multi-échelles de sulfure de zinc par précipitation homogène, PhD Dissertation, Saint-Etienne (France), 2007. 